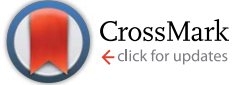

Cite this: RSC Adv., 2016, 6, 50858
Received 8th March 2016

Accepted 16th May 2016

DOI: 10.1039/c6ra06143e

www.rsc.org/advances

\section{Chemical and pharmaceutical evaluation of the relationship between triazole linkers and pore size on cyclodextrin-calixarene nanosponges used as carriers for natural drugs $\dagger$}

\author{
M. Massaro, ${ }^{a}$ V. Cinà, ${ }^{a}$ M. Labbozzetta, ${ }^{b}$ G. Lazzara, ${ }^{c}$ P. Lo Meo, ${ }^{\star a}$ P. Poma, ${ }^{b}$ S. Riela*a \\ and R. Noto ${ }^{a}$
}

Mixed cyclodextrin-calixarene nanosponges were used to prepare some composites with the well known polyphenolic bioactive compounds quercetin and silibinin. The composites were characterized by means of different techniques (UV-vis, FT-IR, microcalorimetry, thermogravimetry), in order to assess their loading and thermal stability. The kinetics of release of the bioactive molecules into aqueous solution were studied at two different $\mathrm{pH}$ values $(1.0,6.4)$, which mimic typical physiological conditions. Finally the possible antiproliferative effects in vitro were assayed towards three triple negative breast cancer cell lines (SUM 149, SUM 159 and MDA-MB-23). Our results point out the role assumed by the triazole linkers, present in the nanosponge network, in affecting both the adsorption abilities of the materials towards the bioactive molecules, and the antiproliferative activity of the composites. This work puts forward an efficient strategy to prepare nanosponge based nanocarriers with three different cavities that could encapsulate two or more drug molecules with different physico-chemical properties.

\section{Introduction}

Natural phenolic compounds such as flavonoids and flavonolignanes are a widespread group of constituents universally distributed among vascular plants. Interest in them has increased in recent years, due to compelling evidence of their health benefits and impact on food quality. Epidemiological studies have shown that flavonoids and flavonolignanes possess anti-oxidant, anti-inflammatory and anti-ageing activities. ${ }^{\mathbf{1}-3}$ Among the phenolic compounds known to date, quercetin (Que) and silibinin (Sil) have attracted great attention (Fig. 1). In particular Que, a flavonoid mainly distributed in fruits, vegetables and teas, ${ }^{4}$ has been shown to have a variety of biological and pharmacological actions, ${ }^{5}$ including decreasing blood lipid levels, ${ }^{6}$ anti-platelet aggregation, ${ }^{7}$ anti-anemic action and antianaphylaxis effects. ${ }^{8}$ Sil is the main flavono-lignan of silymarin, a standardized extract from silybum marianum L.

\footnotetext{
a Dipartimento STEBICEF, Sez. Chimica, Università degli Studi di Palermo, Viale delle Scienze, Parco d'Orleans II, Ed. 17, 90128 Palermo, Italy.E-mail: serena.riela@unipa. it; paolo.lomeo@unipa.it

${ }^{b}$ Dipartimento di Scienze per la Promozione della Salute e Materno Infantile "G. D'Alessandro", Area Funzionale di Farmacologia, Università di Palermo, Via del Vespro 129, 90127 Palermo, Italy

'Dipartimento di Fisica e Chimica, Università degli Studi di Palermo, Viale delle Scienze, Parco d'Orleans II, Ed. 17, 90128 Palermo, Italy

$\dagger$ Electronic supplementary information (ESI) available: ITC and fluorescence titration and thermoanalytical curves, FT-IR spectra. See DOI: 10.1039/c6ra06143e
}

(Asteraceae), used for its hepatoprotective effects. ${ }^{9}$ Its antioxidant and membrane-stabilizing properties are well documented in vitro, being assessed as the main protective mechanisms. ${ }^{10}$ Sil has been reported to possess anti-inflammatory and antifibrotic effects, and to decrease the risk factors of atherosclerosis. ${ }^{\mathbf{1 1}, \mathbf{1 2}}$

There is a growing interest in the potentially beneficial effects on the chemical prophylaxis and therapeutic effects of Que and Sil for cancer treatment. Indeed, many studies have shown that these compounds have anticancer activities against various cancer cells such as colon, prostate, skin, glioma and breast cancer cells. ${ }^{13-15}$ However pharmaceutical applications of phenolic compounds in general are severely hampered by their unsatisfactory bioavailability, due to poor water solubility, low stability and short half-life..$^{15,16}$ In addition, the biological activity of these natural products can be affected by physicochemical factors such as light, oxygen/air, humidity or other reactive compounds from the environment. In order to protect these bioactive compounds against degradation and to enhance their bioavailability, in recent years various encapsulation and complexation methods have been explored. Several possibilities have been successfully tested, in particular nanoparticle composites available in various forms like lipids, ${ }^{17,18}$ biocompatible polymers, ${ }^{19}$ nanotubes,${ }^{20}$ and finally nanosponges.

Nanosponges (NSs) are a new class of materials obtained by polymerizing potential supramolecular hosts with colloidal and nanosized cavities. Their hyper-reticulated three-dimensional 
Que<smiles>O=c1c(O)c(-c2ccc(O)c(O)c2)oc2cc(O)cc(O)c12</smiles>

Sil

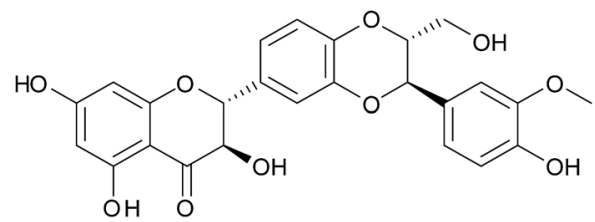

Fig. 1 Structures of quercetin (Que) and silibinin (Sil).

network enables the capture, transportation and prolonged release of a large variety of substances. ${ }^{21}$ The most known examples are the cyclodextrin-based NSs, obtained by reacting native $\beta$-cyclodextrin ( $\boldsymbol{\beta C D}$ ) with double electrophiles such as epichloridrin, bis-isocyanates or carbonic acid derivatives. ${ }^{22,23}$ These materials are biocompatible, cheap, easy to obtain and show several advantageous properties compared to parent cyclodextrin molecules. ${ }^{24}$ In principle, their binding abilities and controlled release properties might be tailored and targeted by means of a suitable choice of the linker. ${ }^{25}$ This distinctive structure organization promotes molecule complexation and might be responsible for the enhanced solubility, dissolution and stabilization of biomolecules ${ }^{\mathbf{2 6 - 2 8}}$ as well as improves the drug bioavailability by modifying the pharmacokinetic parameters of active constituents. ${ }^{29}$ In addition, NSs have the ability to load both hydrophilic and hydrophobic drug molecules because of their inner hydrophobic cavity and external hydrophilic branching, thereby offering unparalleled flexibility. ${ }^{24}$

NSs have already found various applications such as removal of organic pollutant, ${ }^{30,31}$ oxygen and drug delivery systems. ${ }^{32,33}$ In particular, a few recent studies have focused on their inclusion/release properties towards flavonoids such as resveratrol and Que itself. ${ }^{22,30}$

We have recently reported the synthesis of a new class of nanosponges, obtained by co-polymerization of two different supramolecular hosts having potentially complementary binding abilities, namely $\mathbf{B C D}$ and calix[4]arene (CA), covalently linked by means of triazole units. ${ }^{34}$ Different materials have been obtained, with different porosity and hydrophobic properties, by simply changing the co-monomer combination ratio. Tests have positively assessed the actual ability of our materials to sequester organic molecules from an aqueous solution. Moreover, the presence of a triazole linker could play a crucial role in their use as drug carrier. It is known that triazoles possess several interesting biological and pharmaceutical activities such as antibacterial, antifungal, antitumor, plant growth regulating and cytotoxic effects. ${ }^{35-37}$

In the present work we investigated the feasibility of utilizing three $\boldsymbol{B C D}$-CA-NSs, prepared with different co-monomer combination ratios, as possible carrier/delivery systems for Sil and Que. In particular, we explored the supramolecular binding abilities of these materials, and verified whether the biological

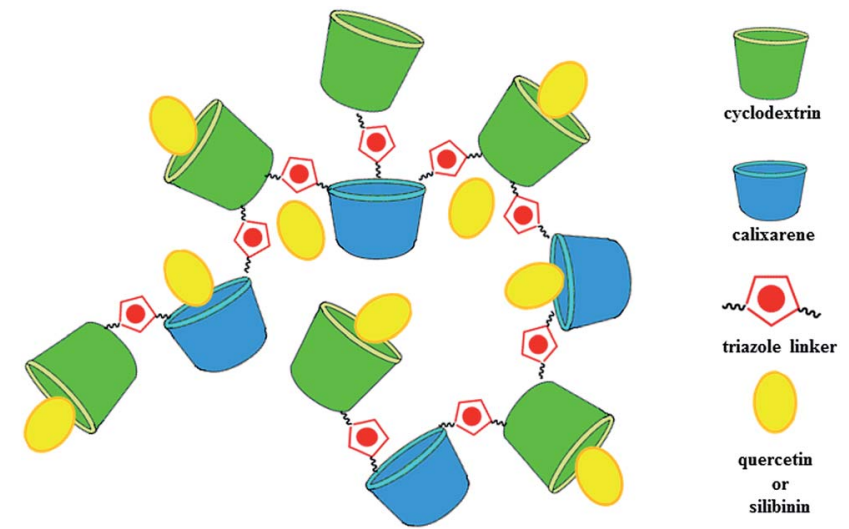

Fig. 2 Cartoon depiction of the composites hypothesized.

activities of Que and Sil are synergistically improved by the possible concomitant cellular uptake of the triazole-containing material. In this study we prepared the six possible Que/NSs and Sil/NSs composites (Fig. 2), which were characterized by means of FT-IR spectroscopy, Isothermal Calorimetric Titration (ITC) and thermogravimetric analysis (TGA). Then, we studied the kinetics of release of the bioactive molecules from the NS composites and their anti-proliferative activity towards three triple negative breast cancer cell lines (namely MDA-MB-231, SUM-149 and SUM-159).

The advantages of nanosponges with "three" different cavities, namely $\mathbf{\beta C D}, \mathbf{C A}$ and nanochannels could offer the remarkable possibility for a simultaneous encapsulation of two or more drug molecules with different physico-chemical properties, followed by a different path release in agreement with the cavity that interacts with the drugs.

\section{Results and discussion}

The preparation of the three different nanosponges was accomplished according to literature reports. ${ }^{34}$ In particular, the NSs were obtained by the CuAAC reaction (Fig. 3) between the heptakis-(6-deoxy)-(6-azido)- $\boldsymbol{\beta C D}$ ( $\boldsymbol{\beta C D A}$ ) and the tetrakis$(25,26,27,28$-propargyloxy)-(5,11,17,23-tert-butyl)-calix[4]arene (CX) in different molar ratio. It is interesting to note that the nanosponges were prepared with an excess of the azide component, that could undergo further functionalizations. Three different polymeric compounds were isolated and their compositions were determined by ${ }^{13} \mathrm{C}\left\{{ }^{1} \mathrm{H}\right\}$ CP-MAS NMR (see Fig. S1 in ESI $\dagger){ }^{34}$ The amount of triazole linkers per $g$ of material, and the average pore size obtained by analysis of the desorption branch using the BJH calculation method (see Fig. S2 in ESI $\dagger$ ), are summarized in Table 1.

It is worth noting that the amount triazole linkers and the porous structure significantly change on increasing the $\boldsymbol{\beta C D A} /$ CX ratio. These parameters are crucial in determining the hostguest interactions and in affecting the biological activity of the new drug carriers. The drug molecules, indeed, may be included into the cavities of both $\boldsymbol{\beta C D A}$ or $\mathbf{C X}$ and, due to crosslinking, simultaneous interaction of the guest molecules with two co- 


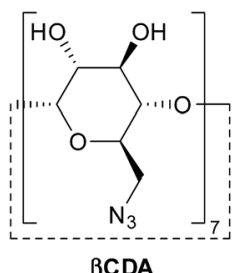

$\beta C D A$

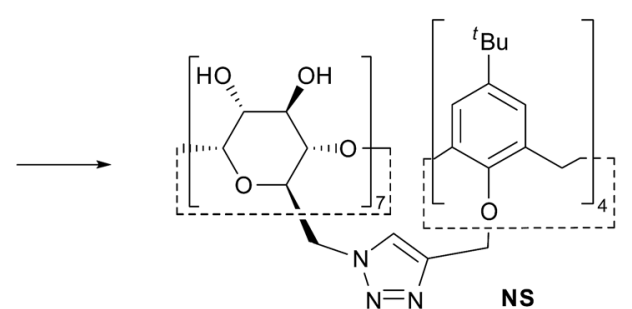

Fig. 3 Synthesis of the nanosponges.

Table 1 Analytical data relevant to NSs

\begin{tabular}{llll}
\hline & $\begin{array}{l}\text { BCDA : CX } \\
\text { mole ratio }\end{array}$ & $\begin{array}{l}\text { Triazole } \\
\text { linkers }\end{array}$ & $\begin{array}{l}\text { Average pore } \\
\text { size }^{b}(\mathrm{~nm})\end{array}$ \\
\hline NS1 & $0.91: 1$ & 1.69 & $3.5 \pm 0.4$ \\
NS2 & $0.57: 1$ & 2.02 & $2.7 \pm 0.3$ \\
NS3 & $0.29: 1$ & 1.46 & $1.9 \pm 0.2$
\end{tabular}

${ }^{a}$ mmoles per $\mathrm{g}$ of material. ${ }^{b}$ See ref. 34 .

monomer subunits units is possible. Moreover, crosslinking may also form nanochannels within the NS structure of the polymer mesh. ${ }^{38}$

\section{Investigations of the binding abilities of the nanosponges in solution}

Previous investigations had shown that native $\boldsymbol{\beta C D}$ and modified $\boldsymbol{\beta C D A}$ can effectively include quercetin, whereas silibinin does not interact with the $\boldsymbol{\beta C D A}^{\mathbf{2 0 , 3 9}}$ In order to verify whether quercetin and silibinin interact with the calixarene cavity, supramolecular interactions with the tetrakis- $(25,26,27,28-((1-$ carboxymethyl)-1H-1,2,3-triazol-4-yl)methoxy)-(5,11,17,23-tertbutyl)-calix[4]arene sodium salt (CS, Fig. 4), chosen to model in solution the calixarene units of the NSs, have been studied. The

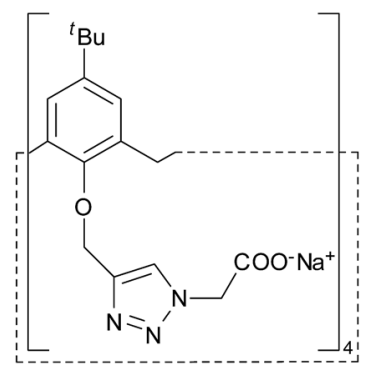

Fig. 4 Structure of the model calixarene CS. formation of 1:2 quercetin/calixarene complexes has been demonstrated by isothermal titration calorimetry (ITC).

The ITC data showed endothermic effects with a sigmoidal profile of the interaction heat vs. Que : CS mole ratio (see Fig. S3 in ESI $\dagger$ ). Data analysis provided the equilibrium constant and the enthalpy change for the quercetin-calixarene inclusion complex formation by assuming the equilibria:

$$
\begin{gathered}
\text { CS }+ \text { Que } \stackrel{K_{1}}{\rightleftharpoons}[\mathbf{C S}-\text { Que }] \\
{[\mathbf{C S}-\text { Que }]+\mathbf{C S} \stackrel{K_{2}}{\rightleftharpoons}[\mathbf{C S}-\text { Que }-\mathbf{C S}]}
\end{gathered}
$$

and the procedure reported elsewhere. ${ }^{40}$

It was found $\beta_{2}=K_{1} K_{2}=(1.4 \pm 0.6) 10^{6} \mathrm{M}^{-2}$ and an enthalpy change $\left(\Delta H^{\circ}\right)$ of $14.8 \pm 0.6 \mathrm{~kJ} \mathrm{~mol}^{-1}$. Then, an entropic contribution $\left(T \Delta S^{\circ}\right)$ as large as $50 \pm 15 \mathrm{~kJ} \mathrm{~mol}^{-1}$ can be computed, highlighting the hydrophobic effect as the driving force for Que incorporation within the calixarene cavity. The silibinin interactions with calixarenes has been reported. ${ }^{\mathbf{4 1}}$

In order to evaluate the binding abilities of the NSs towards Que and Sil, we performed some sequestration texts by mechanically shaking weighed amounts of the NSs in the presence of diluted solutions of Que and Sil at different $\mathrm{pH}$ values (namely 1.0, 4.4 and 6.7). The loading efficiency for each nanosponge was estimated by UV-vis spectrophotometry. Results are represented in Fig. 5. As it can be easily seen, all the nanosponges can absorb both guests in different extent depending on the $\mathrm{pH}$ considered. In particular, silibinin was more efficiently absorbed than quercetin, suggesting the occurrence of stronger supramolecular interactions with the
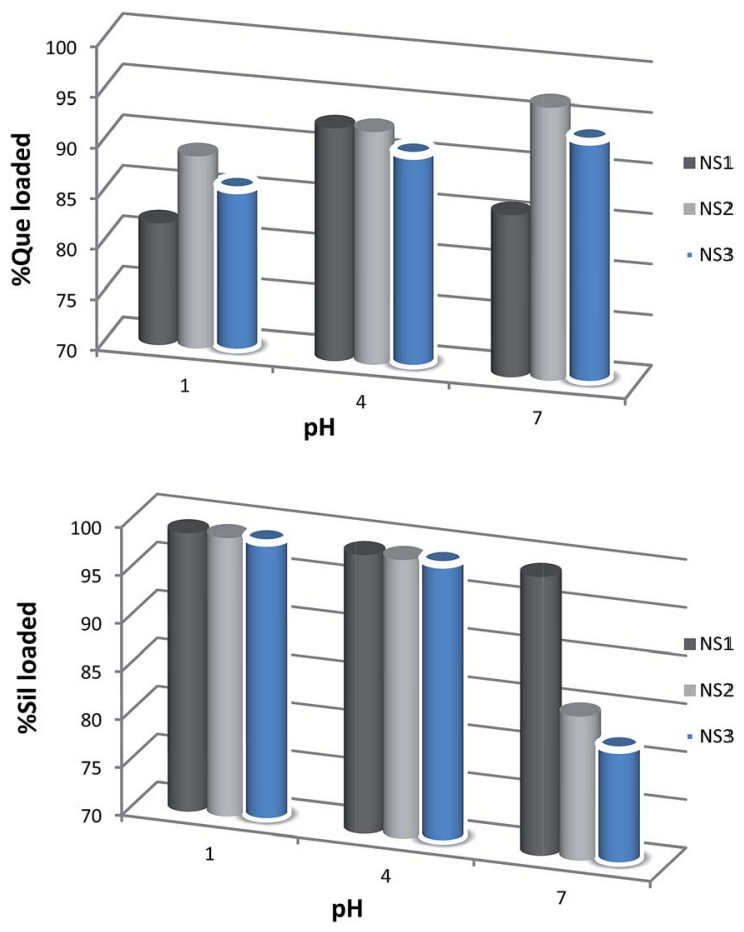

Fig. 5 Amount of Que or Sil loaded in the nanosponges. 
cavities of the NSs. As long as quercetin is concerned, NS2 showed the best binding abilities at each $\mathrm{pH}$. This may be explained by the presence of a larger amount of triazole rings, which enhances the hydrogen bond donor/acceptor abilities of the NS matrix.

Indeed, the importance of hydrogen bonding in affecting the inclusion properties of native $\boldsymbol{\beta C D s}$ has been positively assessed, as it has been previously reported for polyhydroxylated $p$-nitroaniline derivatives..$^{\mathbf{4 2 - 4 4}}$ On the other hand, on increasing the pH NS2 and NS3 showed a decrease in its ability to interact with silibinin. This behavior is probably due to the lesser porosity of the two nanosponges in comparison with the other material (see Table 1). Variations in the porosity of the NSs can be attributed to the cross-linked network, which forms nanochannels in the NS framework. Our data clearly point out that a key factor in controlling the binding abilities of the nanosponges is strictly correlated to the size of the pores, and therefore to the presence of the interstitial channels. Indeed, the formation of nanochannels enables a large amount of drug to be incorporated in the inner structure of the NS.

\section{Investigations of solid state composites}

With the final aim to reach the most effective loading of both drugs in NSs, we decided to perform loading from a solution buffered at pH 4.4. The loading efficiencies of Que/NSs and Sil/ NSs estimated spectrophotometrically after extraction with methanol, are reported in Table 2 as the percent amounts of drug in the final composite and as drug encapsulation efficiency. It is worth noting that data perfectly mirror the results obtained in the aforementioned binding tests. The solid composites were then characterized by means of different techniques.

As a representative example, in Fig. 6 the FT-IR spectrum of Que/NS1 and, for comparison, the spectra of the NS1 and Que are depicted. Our nanosponges are reported to show intense bands in the region around $3300 \mathrm{~cm}^{-1}$, due to the hydroxyl groups of the $\boldsymbol{\beta C D s}$ subunits, and a peak in the region around $2100 \mathrm{~cm}^{-1}$, which can be attributed to the presence of unreacted azide or alkyne groups. Moreover, the fingerprint region shows the presence of signals attributable to both the $\boldsymbol{\beta C D}$ $\left(1295,1156,1078,1049 \mathrm{~cm}^{-1}\right)$ and the $\mathbf{C A}\left(1364\right.$ and $\left.1189 \mathrm{~cm}^{-1}\right)$ scaffolds, the intensity of which changes according to the different composition of the material. Finally, it is possible to identify a tiny band at $1239 \mathrm{~cm}^{-1}$ and a shoulder at $c a .1015$ $\mathrm{cm}^{-1}$ accounting for the formation of the triazole ring linkers. The IR spectra of some flavonoids have been carefully analyzed

Table 2 Percent loading amounts and encapsulation efficiency of Que and Sil in the NSs

\begin{tabular}{|c|c|c|c|c|c|c|}
\hline & \multicolumn{3}{|c|}{ Loading amount (\%) } & \multicolumn{3}{|c|}{$\mathrm{EE}(\%)$} \\
\hline & NS1 & NS2 & NS3 & NS1 & NS2 & NS3 \\
\hline Que & 6 & 7 & 5 & 80 & 93 & 67 \\
\hline Sil & 24 & 10 & 9 & 74 & 32 & 28 \\
\hline
\end{tabular}

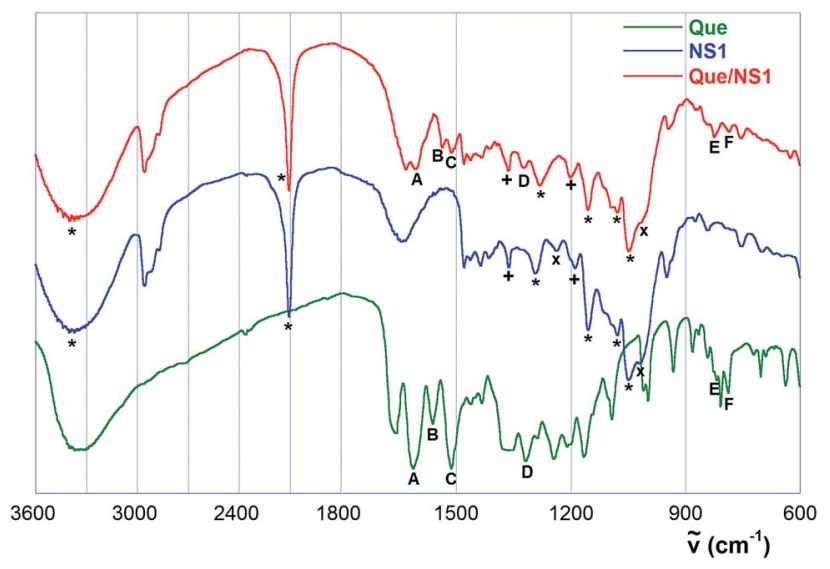

Fig. 6 FT-IR spectra of Que (green), NS1 (blue) and Que/NS1 composite (red). The peaks outlined are relevant to: $(*)$ the CD scaffold; (+) the CA scaffold; $(\times)$ the triazole moiety; $(A-C)$ aromatic Que stretching; (D-F) Que fingerprints.

by Heneczkowski et al., ${ }^{45}$ so the main features in the spectrum of Que are well assessed. In particular, the main signals are due to the stretching of the hydroxyl groups $\left(3336 \mathrm{~cm}^{-1}\right)$ and the carbonyl group $\left(1664 \mathrm{~cm}^{-1}\right)$. Moreover, there are three bands at 1612, 1562 and $1514 \mathrm{~cm}^{-1}$ accounting for aromatic rings vibrations and, among the various signals in the rich fingerprint region, a peak at $1317 \mathrm{~cm}^{-1}$ (attributed to $\mathrm{C}-\mathrm{OH}$ bending) and a peculiar system of three peaks at 820,808 and $788 \mathrm{~cm}^{-1}$.

In the IR spectrum of the composite, the main Que signal at $1664 \mathrm{~cm}^{-1}$ is completely concealed by signals attributable to the polymer matrix (the same occurs for the -OH stretching at 3336 $\mathrm{cm}^{-1}$ ). However, it is possible to notice three peaks at 1606, 1538 and $1514 \mathrm{~cm}^{-1}$ which are clearly due to the presence of the guest and, most important, show significant shifts accounting for a strong interaction between the aromatic rings and the copolymer matrix. Similarly, although other Que fingerprints are hardly visible, a signal at $1323 \mathrm{~cm}^{-1}$ and two further signals at 826 and $787 \mathrm{~cm}^{-1}$ clearly mirror the aforementioned Que peak systems, again with small but significant shifts. The complete collection of FT-IR spectra is reported in ESI (Fig. S4 $\dagger$ ). In brief, spectra of composites NS2/Que and NS3/Que show tiny but clear signals accounting for the presence of the guest at 1606, 1323, 826 and $808 \mathrm{~cm}^{-1}$. A similar analysis can be carried out for the spectra of the NS/Sil composites. Pure Sil has, in particular, a sharp carbonyl band centered at $1638 \mathrm{~cm}^{-1}$, which undergoes a significant enlargement and bathochromic shift in the composites. Among its other fingerprint bands, Sil possesses also a signal at $1510 \mathrm{~cm}^{-1}$ that is apparent also in the composites (slightly shifted up to $1513 \mathrm{~cm}^{-1}$ ), and two tiny signals at 850 and $824 \mathrm{~cm}^{-1}$ respectively, mirrored in the composites by a weak convolution band centered at ca. 834 $\mathrm{cm}^{-1}$.

The thermal stability of the NS/Sil and NS/Que systems was determined by means of TGA. The thermoanalytical curves for pristine components $\mathbf{N S}^{\mathbf{3 4}}$ and $\mathbf{S i l}$ or $\mathbf{Q u e}^{\mathbf{2 0}}$ are multi steps and the mass losses occur in a comparable temperature range. Although the identification of each degradation step in the 
composite material is hardly possible, conclusions on the degradation features of the composites and the effects of each component were straightforward. Fig. 7 shows that the effect of Que and Sil loading on thermal stability was polymer specific. As long as NS1 is concerned, Que and Sil generated an additional mass loss to the pristine NS1 data starting from $500{ }^{\circ} \mathrm{C}$. As both drugs should already degrade below $500{ }^{\circ} \mathrm{C}$ (see ESI, Fig. S5 $\dagger$ ), one may argue that the loading into NS1 strongly enhance the thermal stability of Que and Sil. NS2/drug samples do not show any significant alteration of the TGA profile compared to the pristine NS2 behavior. The case of NS3 is peculiar, because the thermal stability was oppositely influenced by the two drugs. In particular, Que loading enhanced the degradation of the composite while Sil incorporation reduces the mass loss upon heating. In principle the TGA data might be used to calculate the drug loading in a complex mixture, although this is straightforward for inorganic nanocarrier, ${ }^{46}$ it is not generally valid. In fact, it is known that inclusion in macrocycle cavities alters the thermal stability of a compound and the residual charcoal depends on specific interactions, ${ }^{47}$ therefore, rule of mixture is not generally valid as this is clearly the case here (Fig. 5). Notwithstanding, TGA data showed that the amount of CD in the NS is crucial for the Que protection.

This result is consistent with the strong binding between Que and the $\boldsymbol{\beta C D}$ cavity $\left(\beta=1.5 \pm 0.4 \times 10^{6} \mathrm{M}^{-2}\right)$ evidenced by fluorescence experiments (see details in ESI, Fig. S6 $\dagger$ ). In other word, both the $\boldsymbol{\beta C D}$ presence and the larger size of the cavity enhances the thermal protection of the drug.

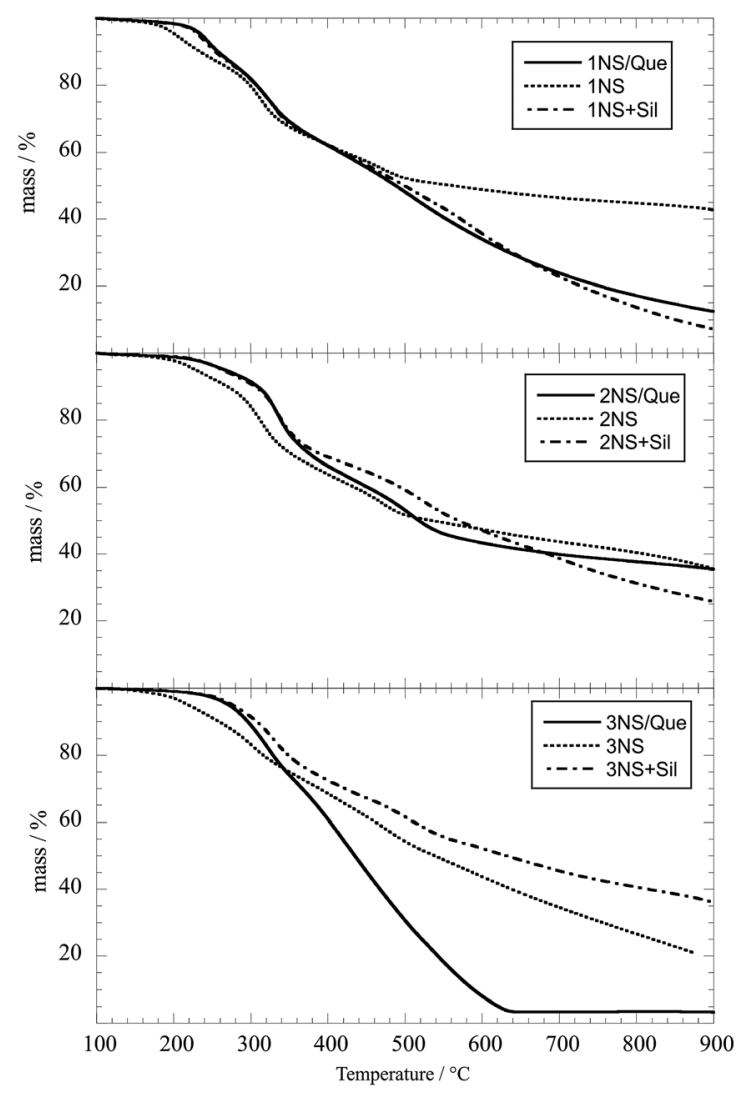

Fig. 7 TGA data for NS and NS/drug complexes.
The controlled release of Que and Sil from the complexes Que/NS and Sil/NS was studied in aqueous buffers at both $\mathrm{pH}$ 1.0 and 6.4 , i.e. in such a way to mimic the typical physiological conditions of either gastric or intestinal fluids. Regarding the quercetin release, it is important to notice that in all the cases considered the spectrum of the released molecule showed a significant bathochromic shift (from 370 to $290 \mathrm{~nm}$ ) of the main UV-vis absorption peak. This shift, however, cannot be attributed to a chemical modification of the Que molecule subsequent to inclusion, due for instance to the unexpected occurrence of oxidation or hydration processes. As a matter of fact, after each release experiment, the residual composite was carefully collected, repeatedly washed with methanol, and the combined methanolic extracts were analyzed by UV-vis, showing the unchanged spectrum of the pure guest. This provides sufficient proof that quercetin undergoes no alteration during the release experiment. By the way, spectrophotometric determination of the residual amount of guest in the composite recovered after the test allowed us to determine the cumulative release. On the other hand, we noticed that, after shaking and equilibrating the pristine nanosponges with the same buffers, the UV-vis spectrum of the aqueous phase shows a broad weak absorption band in the region below $300 \mathrm{~nm}$. This indicates that the nanosponge matrix undergoes slow disgregation, probably due to mechanical shaking. Therefore, it may be reasonably assumed that Que is indeed released into solution as an inclusion complex with the NS fragments. A similar phenomenon has been already noticed in other release systems, ${ }^{48}$ and of course complicates data analysis, because UV-vis absorbances cannot be converted into real concentrations.

As a representative example, cumulative percent release curves for NS1 at both pH 1.0 and 6.4 are shown in Fig. 8. As we can easily see, the $\mathrm{pH}$ conditions have a large outcome on the release. Under acidic conditions, data points can be suitably subjected to fitting analysis by means of an equation of the form:

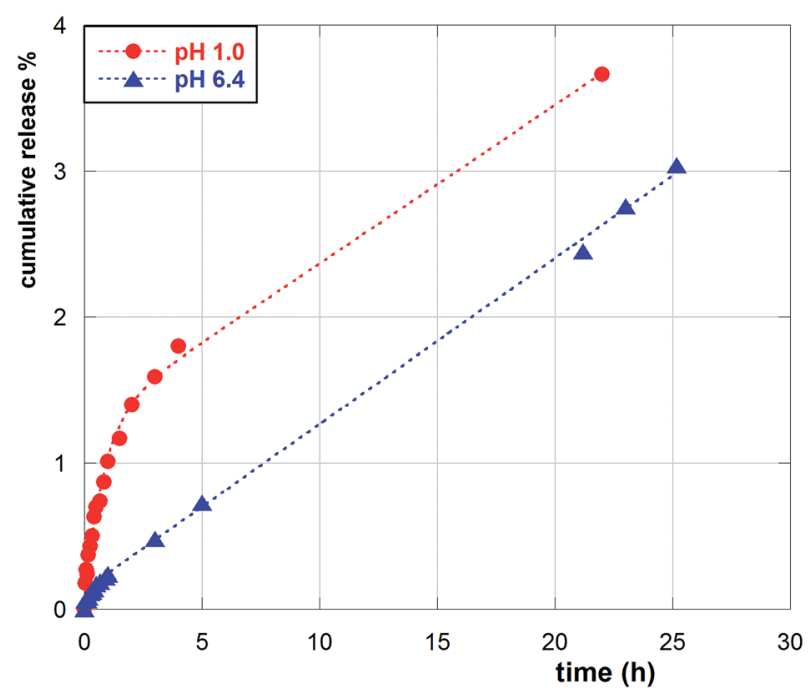

Fig. 8 Kinetics of Que release from NS1 at pH 1.0 and 6.4. 


$$
F_{t}=M_{\infty}\left\lfloor 1-\mathrm{e}^{\left(-k_{1} t\right)}\right\rfloor+k_{0} t
$$

i.e. providing the sum of an exponential and a linear term. This indicates the superimposition of two distinct processes, namely a relatively faster first-order and a zeroth-order ones. By contrast, under nearly neutral conditions the first-order term appears nearly suppressed. The complete values of the apparent kinetic constants $k_{1}$ and $k_{0}$ are summarized in Table 3.

The occurrence of double-term release kinetics is quite common, and may be interpreted in different ways. For instance, it might be due to fast dissolution of the guest absorbed in the more superficial region of the polymer matrix, followed by slower diffusion and release from the inner regions. This hypothesis, however, seems ruled out by the fact that the $\mathrm{pH}$ affects so deeply the kinetic course of the process. More likely, it may outline a release from different (but similarly accessible for the solvent) absorbing sites of the NS. As long as the first-order process is concerned, under acidic conditions, the slower release from Que/NS2 complex seems to parallel the most effective sequestering ability of NS2 found in the binding tests. Once again, this may be interpreted as a consequence of the better ability of NS2 to function as a hydrogen bond donor, consequent to the larger amount of triazole residues present in the NS matrix. On the other hand, under neutral conditions the speed of release seems directly linked to NS porosity.

The $k_{0}$ values relevant to the apparent zeroth-order process, are less easy to interpret. Indeed, these values may be the outcome of a subtle interplay between the actual guest loading, the different composition in co-monomers and the different porosity of the nanosponges. Under acidic conditions, the larger $k_{0}$ value for NS1 can be related to its larger porosity, which counterbalances the hydrogen bond abilities of the protonated triazole units. On the other hand, under neutral conditions, when triazole protonation does not occur, release becomes comparable in the three cases. Therefore, everything considered, the experimental results seem to indicate that the different opposite effects nearly compensate each other under neutral conditions.

Finally, no release of Sil from its composites was observed, probably due to the strong interaction of the molecule with the polymer matrix.

\section{Antiproliferative activity in vitro}

Triple negative breast cancers (TNBCs) are highly aggressive and cytotoxic chemotherapy resistant tumors, thus available treatments are often ineffective. ${ }^{49,50}$

Table 3 Kinetic parameters

\begin{tabular}{llllll}
\hline & $\mathrm{pH} 1.0$ & & & $\mathrm{pH} 6.4$ & \\
\cline { 2 - 3 } \cline { 5 - 6 } Composite & $k_{1} \times 10^{4} \mathrm{~s}^{-1}$ & $k_{0} \times 10^{6} \mathrm{~s}^{-1}$ & & $k_{1} \times 10^{4} \mathrm{~s}^{-1}$ & $k_{0} \times 10^{6} \mathrm{~s}^{-1}$ \\
\hline Que/NS1 & $3.6 \pm 0.4$ & $30.2 \pm 1.5$ & $10 \pm 4$ & $31.5 \pm 0.2$ \\
Que/NS2 & $1.4 \pm 0.6$ & $15.3 \pm 1.8$ & & $1.5 \pm 0.4$ & $30 \pm 3$ \\
Que/NS3 & $3.7 \pm 0.6$ & $20.8 \pm 0.9$ & - & $37.5 \pm 0.6$
\end{tabular}

In order to verify whether the functionalized nanosized composites are good delivery systems of Que and Sil for inhibiting cell proliferation, the cytotoxic effects of the two drugs encapsulated in the NSs, compared to free Que and Sil, were tested on three cell lines of triple negative breast cancer (SUM 149, SUM 159 and MDA-MB-231) by MTS tests. Cell viability was estimated at $72 \mathrm{~h}$ under different concentrations of the compounds (1-100 $\mu \mathrm{M}$ range).

It was observed that cell viability decreases in a concentration and time dependent way (Fig. 9). As expected, the survival rates of the tumor cells incubated with free quercetin and silibinin at each concentration were found in the range of 96$100 \%$, indicating that they have no effect on the viability of the tumor cell lines under the concentration conditions investigated. This is probably due to their insolubility in physiological medium.

It is interesting to notice that each nanosponge showed a cytotoxic effect on the tumoral cell lines investigated; in particular, NS2 seems to be the more active material on all cell lines. This behaviour may be due to the presence of triazole linker in the nanosponge, that it is known to possess very interesting biological activities, in particular antitumoral effect. By contrast, all cell lines showed a dose dependent cytotoxic profile when subjected to the treatment of Que/NS3. This increase in the cytotoxicity of Que/NS3 compared with free quercetin was probably due to the enhanced aqueous solubility of Que/NS3 and to the synergistic effect by the presence of quercetin and triazole. Surprisingly, the composites Que/NS1 and Que/NS2 seemed to be less active in comparison to the free nanosponges. This different behaviour, also in this case, could be due both to the pore dimensions and to the supramolecular interactions between Que with the inner structure of the NS. The Que/NS1-2 composites showed comparable or less antiproliferative activity than the free nanosponges, with greater pore dimensions than NS3, as a consequence of specific $\pi-\pi$ interactions between quercetin and the triazole units present in the interstitial channels that masked the antiproliferative activity of the triazoles themselves. By contrast, the increase in the antiproliferative activity observed for Que/NS3 complex is due, as described above, to a synergistic effect with triazole and

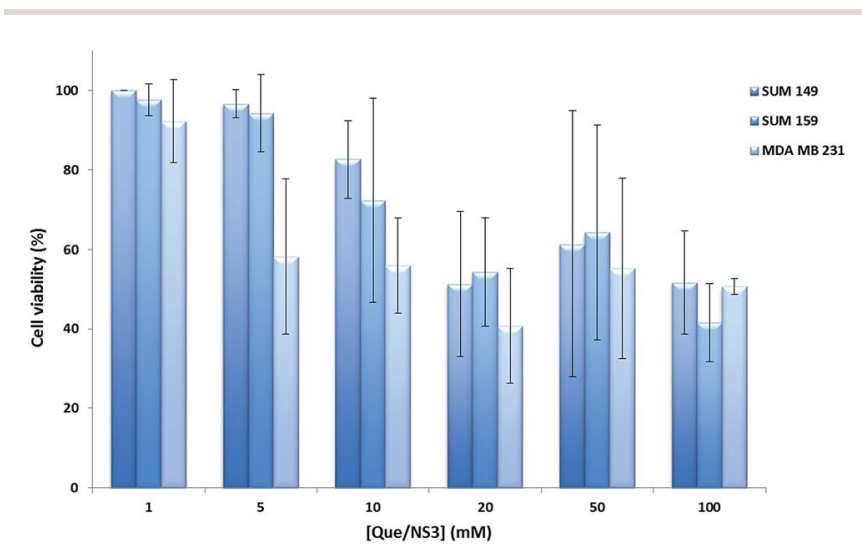

Fig. 9 MTS test on three cell lines of triple negative breast cancer for the Que/NS3 composites. 
Que, that interacted, in this case, predominantly with $\beta$ CDs and calix cavities.

Finally, no cytotoxic effects for the several cell lines in the presence of the complexes Sil/NSs were observed. In our opinion, the availability of silibinin encapsulated in the nanosponges is low, so it is not released in these conditions as highlighted from release studies.

\section{Experimental}

\section{Materials and method}

All needed reagents were used as purchased (Aldrich), without further purification. Nanosponges NS1-NS3 were prepared as previously reported. ${ }^{34}$

Thermogravimetric analyses were performed on a Q5000 IR apparatus (TA Instruments) under a nitrogen flow of $25 \mathrm{~cm}^{3}$ $\mathrm{min}^{-1}$ for the sample and $10 \mathrm{~cm}^{3} \mathrm{~min}^{-1}$ for the balance. The weight of each sample was $c a .10 \mathrm{mg}$. Measurements were carried out by heating the sample after equilibration at $100{ }^{\circ} \mathrm{C}$ up to $900{ }^{\circ} \mathrm{C}$ at a rate of $10{ }^{\circ} \mathrm{C} \mathrm{min}^{-1}$.

FT-IR spectra $(\mathrm{KBr})$ were recorded using an Agilent Technologies Cary 630 FT-IR spectrometer. Specimens for measurements were prepared by mixing $5 \mathrm{mg}$ of the sample powder with $100 \mathrm{mg}$ of $\mathrm{KBr}$.

The ITC experiments were carried out at $25{ }^{\circ} \mathrm{C}$ in a nanoITC200 calorimeter (MicroCal). An amount of approximately $40 \mathrm{~mL}$ of quercetin solution was injected into the cell $(202 \mu \mathrm{L})$ filled with the calix solution. A mixture $\mathrm{H}_{2} \mathrm{O} / \mathrm{MeOH}(6: 4)$ was used as the solvent. The heat effect was measured after each addition of $1 \mu \mathrm{L}$ and corrected by dilution effects.

Synthesis of the model calixarene tetrakis- $(25,26,27,28-((1-$ carboxymethyl)-1H-1,2,3-triazol-4-yl)methoxy)-(5,11,17,23tert-butyl)-calix[4]arene sodium salt (CS)

The tetrakis-(25,26,27,28-propargyloxy)-(5,11,17,23-tert-butyl)calix[4]arene ( $200 \mathrm{mg}, 0.25$ mmoles) was dissolved in $4 \mathrm{~mL}$ of DMSO; then $\mathrm{CuSO}_{4}$ hydrate $(40 \mathrm{mg}, 0.16$ mmoles), sodium ascorbate (62 mg, $0.31 \mathrm{mmoles}$ ) and ethyl azidoacetate $(240 \mathrm{mg}$, 2 mmoles) were added. The mixture, placed in a dark vial, was kept under magnetic stirring at $70{ }^{\circ} \mathrm{C}$ for $18 \mathrm{~h}$. The crude was then poured into water $(50 \mathrm{~mL})$ and centrifuged. The solid residue was dissolved in $5 \mathrm{~mL}$ of warm methanol, then $5 \mathrm{~mL}$ of aqueous $1 \mathrm{M} \mathrm{NaOH}$ were added, and the mixture was stirred overnight. The crude was concentrated in vacuo, re-suspended with $2 \mathrm{~mL}$ of methanol and filtered off. Yield $265 \mathrm{mg}(82 \%)$. IR (nujol): $1625 \mathrm{~cm}^{-1} \cdot{ }^{1} \mathrm{H}$ NMR (DMSO) $\delta$ (ppm): 1.00 (s, 36H, $t \mathrm{Bu}$ ), 3.31 (s, $\left.8 \mathrm{H},-\mathrm{CH}_{2}-\mathrm{COO}-\right), 4.03\left(\mathrm{br} \mathrm{s}, 12 \mathrm{H},-\mathrm{OCH}_{2}-\right.$ overlapped with $\mathrm{Ar}-\mathrm{CH}_{2}-\mathrm{Ar}$ ), 4.25 (br s, $\left.4 \mathrm{H}, \mathrm{Ar}^{-} \mathrm{CH}_{2}-\mathrm{Ar}\right), 6.89$ (s, 8H, Ar-H), 8.13 (s, $4 \mathrm{H}$, triaz-H). ${ }^{13} \mathrm{C}$ NMR $\delta$ (ppm): 31.6, 32.0, 34.2, 61.0, 78.2, 126.1, 129.1, 133.3, 143.8, 146.4, 152.8, 187.2. Elem. anal. found: C 59.41, H 5.66, N 12.96, Na 7.08 (calcd: C 59.44, H 5.61, N 13.00; Na 7.11).

\section{General procedure for the binding tests}

For the preliminary absorption/sequestration tests, stock solutions of the guests $50 \mu \mathrm{M}$ were prepared at the proper $\mathrm{pH}$ value.
A carefully weighed amount $(4 \pm 0.05 \mathrm{mg})$ of nanosponge and 2 $\mathrm{mL}$ of guest solution were mixed in a vial and shaked mechanically at r.t for $90 \mathrm{~min}$ to ensure equilibration. Then, the solution was filtered off with a common $0.45 \mu \mathrm{M}$ Millipore filter. The amount of residual guest was evaluated spectrophotometrically by comparing the absorbances of the filtered solution with the starting stock solution.

\section{General procedure for the nanosponges loading}

A weighed amount $(60 \mathrm{mg})$ of nanosponge was placed in a small beaker and wetted with $1 \mathrm{~mL}$ of methanol; then, $20 \mathrm{~mL}$ of a 0.05 $\mathrm{M}$ phosphate buffer at $\mathrm{pH} 4.4$ were added and the suspension was placed under magnetic stirring. By means of a Chemetrontype micrometric syringe $1 \mathrm{~mL}$ of a $10 \mathrm{mM}$ or $40 \mathrm{mM}$ solution of quercetin and silibinin, respectively, were slowly introduced into the suspension ( $c a .5 \mu \mathrm{L} \mathrm{min}{ }^{-1}$ ), in order to avoid supersaturation of the solution. After completion of the addition, the suspension was kept under stirring overnight; then the solid was centrifugated at $5000 \mathrm{rpm}$ for $15 \mathrm{~min}$, re-suspended in few $\mathrm{mL}$ of water, collected by vacuum filtration, washed with $1 \mathrm{~mL}$ of ice-cold methanol and dried at $50{ }^{\circ} \mathrm{C}$ under vacuum for three hours. Loading was determined as follows. ca. $2 \mathrm{mg}$ of composite were carefully weighed and washed with four portions of methanol ( $2 \mathrm{~mL}$ each). The organic extracts were quantitatively transferred in a volumetric flask $(20 \mathrm{~mL})$ and diluted up to mark with aqueous buffer at $\mathrm{pH} 4.4$; the concentration of the resulting solution was then determined spectrophotometrically.

Drug encapsulation efficiency (EE) was determined with the following equation:

$$
\mathrm{EE}=\left(\frac{\text { actual loading }}{\text { theoretical loading }}\right) \times 100
$$

\section{Kinetic release}

The release of quercetin and silibinin from the Que/NS and Sil/NS composites was performing as follows. $25 \mathrm{mg}$ of the sample were placed in a dialysis membrane (Medicell International Ltd MWCO 12-14 000 with diameter of $21.5 \mathrm{~mm}$ ), wetted with two drops of methanol and dispersed with $1 \mathrm{~mL}$ of the chosen extraction medium. The membrane was then put in a round bottom flask containing $20 \mathrm{~mL}$ of the release medium at $37^{\circ} \mathrm{C}$ under magnetic stirring. Two different media $(0.1 \mathrm{M} \mathrm{HCl}$ and phosphate buffer $\mathrm{pH}$ 6.4, respectively) were considered in order to evaluate the influence of $\mathrm{pH}$ on the release behavior of the drug.

At fixed times, $1 \mathrm{~mL}$ of the release medium was withdrawn and analyzed. To keep constant the volume of the release medium $1 \mathrm{~mL}$ of fresh solution ( $0.1 \mathrm{M} \mathrm{HCl}$, pH 6.4 buffer) was added each time to replace the withdrawn one. After the experiment, the total amount of guest released from the composite was determined by washing repeatedly the collected residue composite with methanol, and determining spectrophotometrically the concentration of the washing solution.

Total amounts of drug released $\left(F_{t}\right)$ were calculated as follows: 


$$
F_{t}=V_{\mathrm{m}} C_{t}+\sum_{i=0}^{t-1} V_{\mathrm{a}} C_{i}
$$

\section{Cell cultures}

MDA MB 231 cell lines were cultured in RPMI 1640, SUM 159 and SUM 149 cell lines were cultured in DMEM/F-12 supplemented with insulin $\left(5 \mu \mathrm{g} \mathrm{mL} \mathrm{mL}^{-1}\right)$. All media were supplemented with $10 \%$ heat-inactivated fetal calf serum, $2 \mathrm{mM}$ L-glutamine, 100 units per $\mathrm{mL}$ penicillin and $100 \mu \mathrm{g} \mathrm{mL}^{-1}$ streptomycin (all reagents were from HyClone Europe Ltd, Cramlington, UK). The cells were cultured in a humidified atmosphere at $37{ }^{\circ} \mathrm{C}$ in $5 \%$ $\mathrm{CO}_{2}$. Cells having a narrow range of passage number were used for all experiments.

\section{MTS assay}

To test the effects of the agents, the cells were seeded at $2 \times 10^{4}$ cells per well onto 96-well plates and then incubated overnight. At time 0 , medium was replaced with fresh complete medium and thereof were added quercetin, NSs, Que/NSs and Sil/NSs in concentrations as indicated. At the end of treatment, $16 \mu \mathrm{L}$ of a commercial solution (obtained from Promega Corporation, Madison, WI, USA) containing 3-(4,5-dimethylthiazol-2-yl)-5-(3carboxymethoxyphenyl)-2-(4-sulphophenyl)-2 $H$-tetrazolium (MTS) and phenazine-ethosulfate were added. The plates were incubated for $2 \mathrm{~h}$ in a humidified atmosphere at $37{ }^{\circ} \mathrm{C}$ in $5 \%$ $\mathrm{CO}_{2}$. The bioreduction of the MTS dye was assessed by measuring the absorbance of each well at $490 \mathrm{~nm}$. Cytotoxicity was expressed as a percentage of the absorbance measured in the control cells.

\section{Conclusions}

Six composites were prepared by including polyphenolic natural compounds quercetin and silibinin in three mixed cyclodextrin-calixarene nanosponges characterized by a different co-monomer composition. Loadings were studied under different $\mathrm{pH}$ conditions and were rationalized in terms of the porosity and the hydrogen bond donor-acceptor abilities of the materials, as well as of the intrinsic affinity of the guest molecule for the cavities of the co-monomers. For the latter purpose, binding in solution with a model calixarene was studied by means of microcalorimetry. The composites were also subjected to spectroscopic (FT-IR) and thermogravimetric investigation, which confirmed the strong interaction of the guest molecules with the polymer matrix. Finally, both release studies at different $\mathrm{pH}$ values and antiproliferative assays in vitro towards tumor cell lines converged in indicating the importance of the porosity of the material and in particular role assumed by the presence of the triazole linkers in affecting the possible exploitation of the composites for clinical applications.

\section{Acknowledgements}

The work was financially supported by the University of Palermo, FIRB 2012 (prot. RBFR12ETL5), PON-TECLA (PON03PE_00214_1).

\section{Notes and references}

1 W.-Y. Huang, Y.-Z. Cai and Y. Zhang, Nutr. Cancer, 2009, 62, 1-20.

2 L. Wang, B. Wang, H. Li, H. Lu, F. Qiu, L. Xiong, Y. Xu, G. Wang, X. Liu, H. Wu and H. Jing, Eur. J. Pharmacol., 2012, 690, 133-141.

3 B. Romano, E. Pagano, V. Montanaro, A. L. Fortunato, N. Milic and F. Borrelli, Phytother. Res., 2013, 27, 1588-1596.

4 P. C. H. Hollman and I. C. W. Arts, J. Sci. Food Agric., 2000, 80, 1081-1093.

5 K. Murota and J. Terao, Arch. Biochem. Biophys., 2003, 417, 12-17.

6 Y. Chen, R. Zheng, Z. Jia and Y. Ju, Free Radicals Biol. Med., 1990, 9, 19-21.

7 J. Duarte, R. Pérez-Palencia, F. Vargas, M. Angeles Ocete, F. Pérez-Vizcaino, A. Zarzuelo and J. Tamargo, Br. J. Pharmacol., 2001, 133, 117-124.

8 R. Bucki, T. J. J. Pastore, F. Giraud, J. C. Sulpicejand and P. A. Janmey, J. Thromb. Haemostasis, 2003, 1, 1820-1828.

9 K. Flora, M. Hahn, H. Rosen and K. Benner, Am. J. Gastroenterol., 1998, 93, 139-143.

10 P. Surai, Antioxidants, 2015, 4, 204.

11 H. Basaga, G. Poli, C. Tekkaya and I. Aras, Cell Biochem. Funct., 1997, 15, 27-33.

12 T. Radjabian and H. Fallah Huseini, Iran. J. Pharmacol. Ther., 2010, 9, 63-67.

13 J. Bosch-Barrera, B. Corominas-Faja, E. Cuyàs, B. MartinCastillo, J. Brunet and J. A. Menendez, Anticancer Res., 2014, 34, 4323-4328.

14 N. Chhabra, S. Buzarbaruah, R. Singh and J. Kaur, Int. J. Nutr., Pharmacol., Neurol. Dis., 2013, 3, 206-218.

15 S. Sapino, E. Ugazio, L. Gastaldi, I. Miletto, G. Berlier, D. Zonari and S. Oliaro-Bosso, Eur. J. Pharm. Biopharm., 2015, 89, 116-125.

16 T. F. Kellici, D. Ntountaniotis, G. Leonis, M. Chatziathanasiadou, A. V. Chatzikonstantinou, J. BeckerBaldus, C. Glaubitz, A. G. Tzakos, K. Viras, P. Chatzigeorgiou, S. Tzimas, E. Kefala, G. Valsami, H. Archontaki, M. G. Papadopoulos and T. Mavromoustakos, Mol. Pharm., 2015, 12, 954-965.

17 R. Angelico, A. Ceglie, P. Sacco, G. Colafemmina, M. Ripoli and A. Mangia, Int. J. Pharm., 2014, 471, 173-181.

18 A. Linkevičiute, A. Misiunas, E. Naujalis and J. Barauskas, Colloids Surf., B, 2015, 128, 296-303.

19 K. Krukiewicz, A. Stokfisz and J. K. Zak, Mater. Sci. Eng., C, 2015, 54, 176-181.

20 M. Massaro, S. Piana, C. G. Colletti, R. Noto, S. Riela, C. Baiamonte, C. Giordano, G. Pizzolanti, G. Cavallaro, S. Milioto and G. Lazzara, J. Mater. Chem. B, 2015, 3, 40744081. 
21 R. Cavalli, F. Trotta and W. Tumiatti, J. Inclusion Phenom. Macrocyclic Chem., 2006, 56, 209-213.

22 S. Anandam and S. Selvamuthukumar, J. Mater. Sci., 2014, 49, 8140-8153.

23 X. Zhu and W. Ping, Spectrochim. Acta, Part A, 2014, 132, 3843.

24 S. Panda, S. Vijayalakshmi, S. Pattnaik and R. P. Swain, Int. J. PharmTech Res., 2015, 8, 213-224.

25 F. Trotta, R. D. Cavalli, W. Tumiatti, O. Zerbinati, C. Roggero and R. Vallero, EP 1786841 A1, 2007.

26 P. Shende, K. Deshmukh, F. Trotta and F. Caldera, Int. J. Pharm., 2013, 456, 95-100.

27 D. Lembo, S. Swaminathan, M. Donalisio, A. Civra, L. Pastero, D. Aquilano, P. Vavia, F. Trotta and R. Cavalli, Int. J. Pharm., 2013, 443, 262-272.

28 S. Swaminathan, L. Pastero, L. Serpe, F. Trotta, P. Vavia, D. Aquilano, M. Trotta, G. Zara and R. Cavalli, Eur. J. Pharm. Biopharm., 2010, 74, 193-201.

29 F. Trotta and R. Cavalli, Compos. Interfaces, 2009, 16, 39-48. 30 K. A. Ansari, P. R. Vavia, F. Trotta and R. Cavalli, AAPS PharmSciTech, 2011, 12, 279-286.

31 F. Trotta, R. Cavalli, K. Martina, M. Biasizzo, J. Vitillo, S. Bordiga, P. Vavia and K. Ansari, J. Inclusion Phenom. Macrocyclic Chem., 2011, 71, 189-194.

32 S. J. Torne, K. A. Ansari, P. R. Vavia, F. Trotta and R. Cavalli, Drug Delivery, 2010, 17, 419-425.

33 S. Selvamuthukumar, S. Anandam, K. Kannan and R. Manavalan, J. Pharm. Pharm. Sci., 2012, 15, 103-111, http://www.cspsCanada.org.

34 P. Lo Meo, G. Lazzara, L. Liotta, S. Riela and R. Noto, Polym. Chem., 2014, 5, 4499-4510.

35 K. Singh, J. Enzyme Inhib. Med. Chem., 2006, 21, 557-562.

36 G. B. Bagihalli, P. G. Avaji, S. A. Patil and P. S. Badami, Eur. J. Med. Chem., 2008, 43, 2639-2649.
37 Z. Rezaei, S. Khabnadideh, K. Pakshir, Z. Hossaini, F. Amiri and E. Assadpour, Eur. J. Med. Chem., 2009, 44, 3064-3067.

38 M. Rao, A. Bajaj, I. Khole, G. Munjapara and F. Trotta, J. Inclusion Phenom. Macrocyclic Chem., 2013, 77, 135-145.

39 C. Jullian, L. Moyano, C. Yañez and C. Olea-Azar, Spectrochim. Acta, Part A, 2007, 67, 230-234.

40 R. De Lisi, G. Lazzara and S. Milioto, Phys. Chem. Chem. Phys., 2011, 13, 12571-12577.

41 P. S. C. La Marca, I. Deidda, M. R. Marabeti, S. Papasergi, C. Scialabba, M. Licciardi, G. Giammona, G. M. L. Consoli, G. Granata, C. Geraci, A. R. Blanco and P. Guarneri, presented in part at the Congresso Scientifico BioTecnologie Ricerca di base interdisciplinare traslazionale in ambito medico $3^{\circ}$ Meeting, 2015.

42 P. Lo Meo, F. D'Anna, S. Riela, M. Gruttadauria and R. Noto, Org. Biomol. Chem., 2003, 1, 1584-1590.

43 P. Lo Meo, F. D'Anna, M. Gruttadauria, S. Riela and R. Noto, Tetrahedron, 2009, 65, 10413-10417.

44 P. Lo Meo, F. D'Anna, M. Gruttadauria, S. Riela and R. Noto, Tetrahedron, 2004, 60, 9099-9111.

45 M. Heneczkowski, M. Kopacz, D. Nowak and A. Kuźniar, Acta Pol. Pharm., 2001, 58, 415-420.

46 M. Massaro, S. Riela, P. Lo Meo, R. Noto, G. Cavallaro, S. Milioto and G. Lazzara, J. Mater. Chem. B, 2014, 2, 77327738.

47 G. Lazzara and S. Milioto, J. Phys. Chem. B, 2008, 112, 1188711895.

48 M. Massaro, C. G. Colletti, R. Noto, S. Riela, P. Poma, S. Guernelli, F. Parisi, S. Milioto and G. Lazzara, Int. J. Pharm., 2015, 478, 476-485.

49 R. Siegel, J. Ma, Z. Zou and A. Jemal, Ca-Cancer J. Clin., 2014, 64, 9-29.

50 L. Hutchinson, Nat. Rev. Clin. Oncol., 2010, 7, 669-670. 